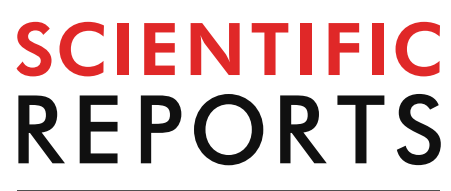

natureresearch

Check for updates

\title{
SNP-SNP interactions of oncogenic long non-coding RNAs HOTAIR and HOTTIP on gastric cancer susceptibility
}

\author{
Esmat Abdi ${ }^{1}$, Saeid Latifi-Navid ${ }^{1 凶}$, Saber Zahri ${ }^{1}$, Vahid Kholghi-Oskooei ${ }^{2,3}$, \\ Behdad Mostafaiy ${ }^{4}$, Abbas Yazdanbod ${ }^{5}$ \& Farhad Pourfarzi ${ }^{5}$
}

Genetic variants within oncogenic long non-coding RNAs HOTAIR and HOTTIP may affect their gene expression levels, thereby modifying genetic susceptibility to gastric cancer (GC). In a hospital-based study in Ardabil-a very high-risk area in North-West Iran, 600 blood samples from 300 GC patients and 300 healthy controls were recruited for genotyping. Seven HOTAIR (i.e., rs17720428, rs7958904, rs1899663, and rs4759314) and HOTTIP (i.e., rs3807598, rs17501292, and rs1859168) 'tag' single nucleotide polymorphisms (SNPs) were genotyped by the Infinium HTS platform. The rs17720428, rs7958904, and rs1899663 tagSNPs significantly increased GC risk under dominant models by 1.5-, 1.57-, and 1.5-fold, respectively. The G-C-T-A haplotype of HOTAIR tagSNPs increased the risk of GC by 1.31-fold. No significant association was found between HOTTIP SNPs and the risk of GC. HOTAIR and HOTTIP variants were also not associated with any clinicopathologic characteristics. The SNP-SNP interaction of HOTAIR rs17720428/rs7958904 with HOTTIP rs1859168 was associated with an increased risk of GC (rs17720428 TG-rs1859168 CC, OR =1.76; rs7958904 GC-rs1859168 CC, OR = 1.85; rs7958904 CC-rs1859168 CC, OR =1.86). Interestingly, the SNP-SNP interaction of HOTAIR rs1899663 with HOTTIP rs1859168 strongly increased the risk of GC (rs1899663 GT-rs1859168 CC, $\mathrm{OR}=4$.3; rs1899663 TT-rs1859168 CC, OR = 9.37; rs1899663 TT-rs1859168 CA, OR =6.59). We showed that the HOTAIR rs17720428, rs7958904, and rs1899663 tagSNPs and their interactions with the HOTTIP rs1859168 polymorphism significantly increased the risk of GC. Specifically, novel SNP-SNP interactions between HOTAIR and HOTTIP tagSNPs have a larger impact than individual SNP effects on GC risk, thereby providing us with valuable information to reveal potential biological mechanisms for developing GC.

Gastric cancer (GC) is a prevalent disease of the digestive system ${ }^{1-3}$. It is the fifth prevalent kind of cancer $(5.7 \%)$ and the third cause of cancer-related mortality $(8.2 \%)^{4}$. In spite of the incidence decline in some parts of the world, GC is a crucial challenge since most incidences are diagnosed at advanced stages, following poor prognosis $^{5,6}$. Thus, reliable biomarkers of GC must be identified for effective therapy, early diagnosis, and prognosis evaluation. Single nucleotide polymorphisms (SNPs) have profound to have influences on gene function and expression, and contribute to carcinogenesis. Studies of genome-wide association which scan the whole genome for prevalent genetic variants have shown over 450 SNPs related to susceptibility to different cancer types ${ }^{7}$. Only $7 \%$ of these loci are in protein-coding areas, but $93 \%$ are located in non-coding areas ${ }^{8,9}$. Non-coding RNAs are the major regulators of some biological processes, including translation, transcription, epigenetic gene expression, splicing, cell cycle, embryogenesis, stem cell pluripotency and reprogramming, and the immune response regulation ${ }^{10,11}$. Aberrant expression of long non-coding RNAs (lncRNAs) may bring about different cancers ${ }^{12-14}$.

\footnotetext{
${ }^{1}$ Department of Biology, Faculty of Sciences, University of Mohaghegh Ardabili, 5619911367 Ardabil, Iran. ${ }^{2}$ Department of Laboratory Sciences, School of Paramedical Sciences, Torbat Heydariyeh University of Medical Sciences, 9516915169 Torbat Heydariyeh, Iran. ${ }^{3}$ Health Sciences Research Center, Torbat Heydariyeh University of Medical Sciences, 9516915169 Torbat Heydariyeh, Iran. ${ }^{4}$ Department of Statistics, Faculty of Sciences, University of Mohaghegh Ardabili, 5619911367 Ardabil, Iran. ${ }^{5}$ Digestive Disease Research Center, Ardabil University of Medical Sciences, 5618953141 Ardabil, Iran.『email: s_latifi@uma.ac.ir
} 


\begin{tabular}{|l|l|l|l|l|l|l|l|}
\hline & rs17720428 & rs7958904 & rs1899663 & rs4759314 & rs3807598 & rs17501292 & rs1859168 \\
\hline All subjects & 0.37 & 0.87 & 0.73 & 1 & 0.12 & 0.06 & 0.43 \\
\hline Controls & 0.61 & 0.4 & 0.61 & 1 & 0.56 & 0.06 & 0.36 \\
\hline Patients & 0.23 & 0.56 & 0.23 & 1 & 0.11 & 0.07 & 0.51 \\
\hline
\end{tabular}

Table 1. Exact test for Hardy-Weinberg equilibrium ( $p$-value).

Various lncRNAs are associated with different cancer types ${ }^{15-19}$. HOX transcript antisense RNA (HOTAIR)-a well-known oncogenic lncRNA-is highly expressed in GC tissues and has been recognized as a critical prognostic biomarker for major cancers, including GC. HOTAIR inhibition not only reduces tumor invasiveness but also reverses EMT in GC cells by regulating N-cadherin, E-cadherin, vimentin, and a transcription factor snail. HOTAIR targets miR-126 to activate the multidrug resistance-associated protein 1/phosphatidylinositol 3-kinase (PI3-K)/Akt and thus promotes cisplatin resistance in GC. Specifically, it directly inhibits miR126, promoting the expression of PI3-K regulatory subunit beta and vascular endothelial growth factor A. Therefore, HOTAIR-targeted therapies may potentially improve prognosis and survival of patients suffering from $\mathrm{GC}^{15,20-22}$. The HOXA transcript at the distal tip (HOTTIP), transcribed from the $5^{\prime}$ tip of HOXA cluster, is a cancer-related lncRNA ${ }^{23,24}$. Recruiting HOXA13-HOTAIR and HOXA13-HOTTIP to different sites in the promoter of bone morphogenetic protein 7 (BMP7) is critical for the oncogenic fate of the human gastric cells ${ }^{24}$. HOTTIP was overexpressed significantly in cell lines of GC; HOTTIP down-regulation would hinder cell proliferation, degrade cell invasion and migration, and develop cell apoptosis ${ }^{25}$. Ardabil Province is a very high-risk area in North-West Iran (ASRs, 51.8/100,000 and 24.9/100,000 for males and females, respectively), with one of the highest cardia GC (CGC) rates worldwide. Hence, in a case-control study from Ardabil, we genotyped seven HOTAIR (i.e., rs17720428, rs7958904, rs1899663, and rs4759314) and HOTTIP (i.e., rs3807598, rs17501292, and rs185916) tagSNPs to assess their associations with the risk of GC. In addition, to perform data mining regarding the SNPSNP interactions, all possible pair combinations between all of the HOTAIR and HOTTIP SNPs in relation to GC susceptibility were analyzed.

\section{Results}

General characteristics of the study subjects. Each of GC and control groups consisted of 300 subjects, of whom $74.7 \%$ were males. The average age (mean \pm SD (min-max)) was $66.54 \pm 10.43(34-88)$ and $66.48 \pm 9.71$ (38-91) years for cases and controls, respectively. Age and gender were not significantly different between the case and control groups $(p=0.37$ and $p=1.00$, respectively), which indicated that these groups were matched well with respect to these parameters. The genotype distributions of SNPs in cases and controls met the HardyWeinberg equilibrium conditions (Table 1(. The prevalence rate of GC patients based on the anatomic site of the tumor origin was $52.5 \%$ with CGC, $35 \%$ with NCGC, and $12.5 \%$ with both CGC and NCGC. According to histopathologic features, the prevalence rate of the intestinal-, diffuse-, and indeterminate-type GC was $49.7 \%$, $19.7 \%$, and $30.6 \%$, respectively. Moreover, the $0.8,5.2,45.5$ and $48.5 \%$ of patients were diagnosed at stages I, II, III, and IV, respectively. The clinical and demographic characteristics of the subjects are presented in Table 2.

The association between HOTAIR/HOTTIP tagSNPs and GC risk. The average call rate for the 600 analyzed samples was $99.84 \%$, showing high call rates and high reproducibility. Three SNPs of HOTAIR (i.e., rs17720428; rs7958904; rs1899663) were associated with an increased risk of GC. It was found that the rs17720428 polymorphism was associated with the risk of GC, assuming allelic, dominant, and log-additive models of inheritance. The findings revealed that the rs $17720428 \mathrm{G}$ allele was significantly associated with the increased risk of $\mathrm{GC}$ ( $\mathrm{G}$ vs. $\mathrm{T}, \mathrm{OR}=1.27,95 \% \mathrm{CI}=1.01-1.61 ; p=0.04)$. In the dominant model, subjects carrying the TG + GG genotype of rs17720428, as compared with those carrying the TT genotype, had a significantly higher risk of GC (OR=1.5, 95\% CI: $1.08-2.1 ; p=0.01)$.

The rs7958904 SNP was associated with the risk of GC in allelic, co-dominant, dominant, and log-additive models of inheritance. The rs7958904 C allele was significantly associated with the increased risk of GC (C vs. $\mathrm{G}, \mathrm{OR}=1.31,95 \% \mathrm{CI}: 1.04-1.65 ; p=0.02)$. Subjects carrying the CC or GC genotype of rs7958904, as compared with those carrying the GG genotype in the co-dominant model, showed an increased risk of GC (CC vs. GG, $\mathrm{OR}=1.54,95 \% \mathrm{CI}: 1.07-2.22$ and GC vs. GG, OR=1.64, 95\% CI: $1.03-2.62 ; p=0.04)$. In the dominant model, subjects carrying the GC + CC genotype of rs7958904 showed an increased risk of GC in comparison with those carrying the GG genotype (OR=1.57, 95\% CI: $1.1-2.22 ; p=0.01)$.

The rs 1899663 SNP was associated with the risk of GC, assuming allelic, dominant and log-additive models of inheritance. The findings indicated that the rs1899663 $\mathrm{T}$ allele was significantly associated with the increased risk of GC (T vs. G, OR=1.27, 95\% CI: 1.01-1.61; $p=0.04$ ). The GT + TT genotype of rs 1899663 , in comparison with GG genotype, had a significantly higher risk of GC in the dominant model (OR $=1.5,95 \% \mathrm{CI}$ : $1.08-2.08 ; p=0.02)$. No significant associations were observed between the rs4759314 SNP and GC susceptibility (Table 3). No evidence regarding the association between the HOTTIP tagSNPs (i.e., rs3807598, rs17501292, and rs1859168) and GC risk was found in any of the genetic models ( $p>0.05$; Table 4$)$. The HOTAIR and HOTTIP variants were not associated with any clinicopathologic characteristics (Table 5). Moreover, the frequency of each HOTAIR/HOTTIP tagSNP did not show a significant difference between patients having stage I-II and stage III-IV disease (Table 6). 


\begin{tabular}{|c|c|c|}
\hline \multicolumn{3}{|l|}{ Case } \\
\hline Age $($ mean \pm SD $(\min -\max ))$ & - & $66.54 \pm 10.43(34-88)$ \\
\hline \multirow{2}{*}{ Gender } & Male & $74.7 \%$ \\
\hline & Female & $25.3 \%$ \\
\hline \multirow[t]{3}{*}{ Tumor origin } & Cardia & $52.5 \%$ \\
\hline & Cardia and non-cardia & $12.5 \%$ \\
\hline & Non-cardia & $35 \%$ \\
\hline \multirow[t]{3}{*}{ Pathology } & Intestinal-type GC & $49.7 \%$ \\
\hline & Diffuse-type GC & $19.7 \%$ \\
\hline & Other & $30.6 \%$ \\
\hline \multirow{4}{*}{ TNM Stage } & I & $0.8 \%$ \\
\hline & II & $5.2 \%$ \\
\hline & III & $45.5 \%$ \\
\hline & IV & $48.5 \%$ \\
\hline \multirow{4}{*}{$\mathrm{T}$} & $\mathrm{T} 1$ & $9.4 \%$ \\
\hline & $\mathrm{T} 2$ & $18.1 \%$ \\
\hline & T3 & $24.2 \%$ \\
\hline & $\mathrm{T} 4$ & $48.3 \%$ \\
\hline \multirow{4}{*}{$\mathrm{N}$} & N0 & $1.1 \%$ \\
\hline & $\mathrm{N} 1$ & $2.6 \%$ \\
\hline & $\mathrm{N} 2$ & $35.3 \%$ \\
\hline & N3 & $60.9 \%$ \\
\hline \multirow{2}{*}{ M } & M0 & $51.9 \%$ \\
\hline & M1 & $48.1 \%$ \\
\hline \multicolumn{3}{|l|}{ Control } \\
\hline Age $(\operatorname{mean} \pm \mathrm{SD}(\min -\max ))$ & - & $66.48 \pm 9.71(38-91)$ \\
\hline \multirow{2}{*}{ Gender } & Male & 74.7 \\
\hline & Female & 25.3 \\
\hline
\end{tabular}

Table 2. Baseline characteristics of total $300 \mathrm{GC}$ patients and 300 cancer-free controls.

The association of haplotype in two IncRNA genes with GC risk. According to Table 7, the results of haplotype analysis showed that the G-C-T-A haplotype of HOTAIR rs17720428, rs7958904, rs1899663, and rs4759314, respectively, increased the risk of GC by 1.31 -fold (95\% CI: $1.03-1.67 ; p=0.029)$. No haplotype of the three HOTTIP tagSNPs was associated with the risk of GC $(p>0.05)$.

SNP-SNP interaction models for IncRNA polymorphisms. To perform data mining regarding the SNP-SNP interactions, all possible pair combinations between all of the HOTAIR and HOTTIP tagSNPs were analyzed. The interaction of HOTAIR rs17720428 TG with HOTTIP rs1859168 CC potentially increased the risk of GC $(\mathrm{OR}=1.76,95 \% \mathrm{CI}: 1.22-2.54 ; p=0.003)$. In addition, the interaction of HOTAIR rs7958904 with HOTTIP rs1859168 potentially increased the risk of GC (rs7958904 GC-rs1859168 CC, OR=1.85, 95\% CI: $1.25-2.73, p=0.002$; rs7958904 CC-rs1859168 CC, $\mathrm{OR}=1.86,95 \% \mathrm{CI}: 1.14-3.06, p=0.01$ ). Interestingly, the interaction of HOTAIR rs1899663 with HOTTIP rs1859168 strongly increased the risk of GC (rs1899663 GT-rs1859168 CC, OR=4.3, 95\% CI: 2.75-6.7; rs1899663 TT-rs1859168 CC, OR=9.37, 95\% CI: 5.43-16.18; rs1899663 TT-rs1859168 CA, OR=6.59, 95\% CI: 2.12-20.51; all the $p$-values were $<0.001$.) (Table 8).

The potential impact of each SNP on the establishment or destruction of the miRNA binding site. Bioinformatic analysis showed that the HOTAIR rs17720428/rs7958904 and HOTTIP rs17501292 tagSNPs cause miRNA target gain and loss. Moreover, the HOTTIP rs 1859168 polymorphism could lead to miRNA target gain while the rs3807598 polymorphism could lead to miRNA target loss. For the HOTAIR rs1899663 and rs4759314 tagSNPs, no miRNA target gain or loss was recognized (Table 9).

\section{Discussion}

Evidences have demonstrated that the aberrant expression of lncRNAs may develop various malignancies ${ }^{26,27}$. Moreover, polymorphisms in lncRNAs may influence their expression and bring about GC susceptibility ${ }^{28,29}$. SNPs in lncRNAs may affect different biological processes through affecting biological pathways. Studies have confirmed the roles of lncRNAs as critical regulators of tumorigenesis ${ }^{30}$. The current study explored whether the tagSNPs of HOTAIR (i.e., rs17720428, rs7958904, rs1899663, and rs4759314) and HOTTIP (i.e., rs3807598, rs17501292, and rs185916) affect GC development. The G allele and TG + GG genotype of rs17720428 in HOTAIR significantly increased the risk of $\mathrm{GC}$ ( $\mathrm{G}$ vs. $\mathrm{T}, \mathrm{OR}=1.27$; $\mathrm{TG}+\mathrm{GG}$ vs. TT, $\mathrm{OR}=1.5$, respectively). We also showed 


\begin{tabular}{|c|c|c|c|c|c|c|}
\hline Locus & Model & Genotype & Patients & Controls & Odds Ratio & $p$-value \\
\hline \multicolumn{7}{|c|}{ rs17720428 } \\
\hline & \multirow{2}{*}{ Allele } & $\mathrm{T}$ & $359(59.8 \%)$ & $393(65.5 \%)$ & 1 & 0.04 \\
\hline & & G & $241(40.21 \%)$ & $207(34.5 \%)$ & $1.27(1.01-1.61)$ & \\
\hline & \multirow{3}{*}{ Codominant } & TT & $102(34 \%)$ & $131(43.7 \%)$ & 1 & 0.05 \\
\hline & & TG & $155(51.7 \%)$ & $131(43.7 \%)$ & $1.52(1.07-2.15)$ & \\
\hline & & GG & $43(14.3 \%)$ & $38(12.6 \%)$ & $1.45(0.87-2.41)$ & \\
\hline & \multirow{2}{*}{ Dominant } & TT & $102(34 \%)$ & $131(43.7 \%)$ & 1 & 0.01 \\
\hline & & $\mathrm{TG}+\mathrm{GG}$ & $198(66 \%)$ & $169(56.3 \%)$ & $1.5(1.08-2.1)$ & \\
\hline & \multirow{2}{*}{ Recessive } & $\mathrm{TT}+\mathrm{TG}$ & $257(85.7 \%)$ & $262(87.4 \%)$ & 1 & 0.55 \\
\hline & & GG & $43(14.3 \%)$ & $38(12.6 \%)$ & $1.15(0.72-1.84)$ & \\
\hline & \multirow{2}{*}{ Overdominant } & $\mathrm{TT}+\mathrm{GG}$ & $145(48.3 \%)$ & $169(56.3 \%)$ & 1 & 0.05 \\
\hline & & TG & $155(51.7 \%)$ & $131(43.7 \%)$ & $1.38(1-1.9)$ & \\
\hline & Log-Additive & & & & $1.28(1.01-1.63)$ & 0.04 \\
\hline \multicolumn{7}{|c|}{ rs7958904 } \\
\hline & \multirow{2}{*}{ Allele } & G & $315(52.7 \%)$ & $355(59.4 \%)$ & 1 & 0.02 \\
\hline & & $\mathrm{C}$ & $283(47.3 \%)$ & $243(40.6 \%)$ & $1.31(1.04-1.65)$ & \\
\hline & \multirow{3}{*}{ Codominant } & GG & $80(26.8 \%)$ & $109(36.5 \%)$ & 1 & 0.04 \\
\hline & & GC & $155(51.8 \%)$ & $137(45.8 \%)$ & $1.54(1.07-2.22)$ & \\
\hline & & $\mathrm{CC}$ & $64(21.4 \%)$ & $53(17.7 \%)$ & $1.64(1.03-2.62)$ & \\
\hline & \multirow{2}{*}{ Dominant } & GG & $80(26.8 \%)$ & $109(36.5 \%)$ & 1 & 0.01 \\
\hline & & $\mathrm{GC}+\mathrm{CC}$ & $219(73.2 \%)$ & $190(63.5 \%)$ & $1.57(1.1-2.22)$ & \\
\hline & \multirow{2}{*}{ Recessive } & $\mathrm{GG}+\mathrm{GC}$ & $235(78.6 \%)$ & $246(82.3 \%)$ & 1 & 0.26 \\
\hline & & $\mathrm{CC}$ & $64(21.4 \%)$ & $53(17.7 \%)$ & $1.26(0.84-1.9)$ & \\
\hline & \multirow{2}{*}{ Overdominant } & $\mathrm{GG}+\mathrm{CC}$ & $144(48.2 \%)$ & $162(54.2 \%)$ & 1 & 0.14 \\
\hline & & GC & $155(51.8 \%)$ & $137(45.8 \%)$ & $1.27(0.92-1.75)$ & \\
\hline & Log-Additive & & & & $1.31(1.04-1.65)$ & 0.02 \\
\hline \multicolumn{7}{|c|}{ rs1899663 } \\
\hline & \multirow{2}{*}{ Allele } & G & $358(59.9 \%)$ & $393(65.5 \%)$ & 1 & 0.04 \\
\hline & & $\mathrm{T}$ & $240(40.1 \%)$ & $207(34.5 \%)$ & $1.27(1.01-1.61)$ & \\
\hline & \multirow{3}{*}{ Codominant } & GG & $102(34.1 \%)$ & $131(43.7 \%)$ & 1 & 0.06 \\
\hline & & GT & $154(51.5 \%)$ & $131(43.7 \%)$ & $1.51(1.06-2.13)$ & \\
\hline & & TT & $43(14.4 \%)$ & $38(12.7 \%)$ & $1.45(0.87-2.41)$ & \\
\hline & \multirow{2}{*}{ Dominant } & GG & $102(34.1 \%)$ & $131(43.7 \%)$ & 1 & 0.02 \\
\hline & & $\mathrm{GT}+\mathrm{TT}$ & $197(65.9 \%)$ & $169(56.3 \%)$ & $1.5(1.08-2.08)$ & \\
\hline & \multirow{2}{*}{ Recessive } & $\mathrm{GG}+\mathrm{GT}$ & $256(85.6 \%)$ & $262(87.3 \%)$ & 1 & 0.54 \\
\hline & & $\mathrm{TT}$ & $43(14.4 \%)$ & $38(12.7 \%)$ & $1.16(0.72-1.85)$ & \\
\hline & \multirow{2}{*}{ Overdominant } & $\mathrm{GG}+\mathrm{TT}$ & $145(48.5 \%)$ & $169(56.3 \%)$ & 1 & 0.05 \\
\hline & & GT & $154(51.5 \%)$ & $131(43.7 \%)$ & $1.37(0.99-1.89)$ & \\
\hline & Log-Additive & & & & $1.28(1.01-1.62)$ & 0.04 \\
\hline \multicolumn{7}{|c|}{ rs4759314 } \\
\hline & \multirow{2}{*}{ Allele } & A & $586(97.7 \%)$ & $590(98.3 \%)$ & 1 & 0.41 \\
\hline & & G & $14(2.3 \%)$ & $10(1.7 \%)$ & $1.41(0.62-3.2)$ & \\
\hline & & AA & $286(95.3 \%)$ & $290(96.7 \%)$ & 1 & 0.4 \\
\hline & Codominant & AG & $14(4.7 \%)$ & $10(3.3 \%)$ & $1.42(0.62-3.25)$ & \\
\hline & & GG & - & - & - & \\
\hline & Dominant & - & - & - & - & \\
\hline & D & - & - & - & - & \\
\hline & Recessive & - & - & - & - & \\
\hline & Recessive & - & - & - & - & \\
\hline & Overdominant & - & - & - & - & \\
\hline & Overcommant & - & - & - & - & \\
\hline
\end{tabular}

Table 3. Genotype and allele frequencies of HOTAIR SNPs in cases and controls, and genotype- and allelotype-specific risks. OR, odds ratio; CI, confidence interval; SNPs, single nucleotide polymorphisms. 


\begin{tabular}{|c|c|c|c|c|c|c|}
\hline Locus & Model & Genotype & Patients & Controls & Odds Ratio & $p$-value \\
\hline \multicolumn{7}{|c|}{ rs3807598 } \\
\hline & \multirow{2}{*}{ Allele } & $\mathrm{C}$ & $315(52.7 \%)$ & $308(51.5 \%)$ & 1 & 0.68 \\
\hline & & G & $283(47.3 \%)$ & $290(48.5 \%)$ & $0.95(0.76-1.2)$ & \\
\hline & \multirow{3}{*}{ Codominant } & $\mathrm{CC}$ & $90(30.1 \%)$ & $82(27.4 \%)$ & 1 & 0.71 \\
\hline & & CG & $135(45.2 \%)$ & $144(48.2 \%)$ & $0.85(0.58-1.24)$ & \\
\hline & & GG & $74(24.7 \%)$ & $73(24.4 \%)$ & $0.92(0.59-1.35)$ & \\
\hline & \multirow{2}{*}{ Dominant } & $\mathrm{CC}$ & $90(30.1 \%)$ & $82(27.4 \%)$ & 1 & 0.47 \\
\hline & & $\mathrm{CG}+\mathrm{GG}$ & $209(69.9 \%)$ & $217(72.6 \%)$ & $0.89(0.61-1.25)$ & \\
\hline & \multirow{2}{*}{ Recessive } & $\mathrm{CC}+\mathrm{CG}$ & $225(75.3 \%)$ & $226(75.6 \%)$ & 1 & 0.92 \\
\hline & & GG & $74(24.7 \%)$ & $73(24.4 \%)$ & $1.02(0.7-1.48)$ & \\
\hline & \multirow{2}{*}{ Overdominant } & $\mathrm{CC}+\mathrm{GG}$ & $164(54.8 \%)$ & $155(51.8 \%)$ & 1 & 0.46 \\
\hline & & CG & $135(45.2 \%)$ & $144(48.2 \%)$ & $0.89(0.64-1.22)$ & \\
\hline & Log-Additive & & & & $0.96(0.77-1.19)$ & 0.69 \\
\hline \multicolumn{7}{|c|}{ rs17501292 } \\
\hline & \multirow{2}{*}{ Allele } & $\mathrm{T}$ & $492(0.83)$ & $514(0.86)$ & 1 & 0.1 \\
\hline & & G & $104(0.17)$ & $84(0.14)$ & $1.29(0.95-1.77)$ & \\
\hline & \multirow{3}{*}{ Codominant } & TT & $208(69.8 \%)$ & $222(74.3 \%)$ & 1 & 0.29 \\
\hline & & TG & $76(25.5 \%)$ & $70(23.4 \%)$ & $1.16(0.8-1.69)$ & \\
\hline & & GG & $14(4.7 \%)$ & $7(2.3 \%)$ & $2.13(0.84-5.39)$ & \\
\hline & \multirow{2}{*}{ Dominant } & TT & $208(69.8 \%)$ & $222(74.3 \%)$ & 1 & 0.23 \\
\hline & & $\mathrm{TG}+\mathrm{GG}$ & $90(30.2 \%)$ & $77(25.7)$ & $1.24(0.87-1.78)$ & \\
\hline & \multirow{2}{*}{ Recessive } & $\mathrm{TT}+\mathrm{TG}$ & $284(95.3 \%)$ & $292(97.7 \%)$ & 1 & 0.12 \\
\hline & & GG & $14(4.7 \%)$ & $7(2.3 \%)$ & $2.06(0.82-5.17)$ & \\
\hline & \multirow{2}{*}{ Overdominant } & $\mathrm{TT}+\mathrm{GG}$ & $222(74.5 \%)$ & $229(76.6 \%)$ & 1 & 0.55 \\
\hline & & TG & $76(25.5 \%)$ & $70(23.4 \%)$ & $1.12(0.77-1.63)$ & \\
\hline & Log-Additive & & & & $1.27(0.94-1.72)$ & 0.12 \\
\hline \multicolumn{7}{|c|}{ rs1859168 } \\
\hline & \multirow{2}{*}{ Allele } & $\mathrm{C}$ & $569(95.2 \%)$ & $561(93.5 \%)$ & 1 & 0.22 \\
\hline & & A & $29(4.8 \%)$ & $39(6.5 \%)$ & $0.73(0.45-1.2)$ & \\
\hline & \multirow{3}{*}{ Codominant } & $\mathrm{CC}$ & $271(90.7 \%)$ & $263(87.7 \%)$ & 1 & 0.48 \\
\hline & & $\mathrm{CA}$ & $27(9 \%)$ & $35(11.7 \%)$ & $0.75(0.44-1.27)$ & \\
\hline & & $\mathrm{AA}$ & $1(0.3 \%)$ & $2(0.6 \%)$ & $0.48(0.04-5.38)$ & \\
\hline & \multirow{2}{*}{ Dominant } & $\mathrm{CC}$ & $271(90.7 \%)$ & $263(87.7 \%)$ & 1 & 0.24 \\
\hline & & $\mathrm{CA}+\mathrm{AA}$ & $28(9.3 \%)$ & $37(12.3 \%)$ & $0.73(0.48-1.23)$ & \\
\hline & \multirow{2}{*}{ Recessive } & $\mathrm{CC}+\mathrm{CA}$ & $298(90.7 \%)$ & $298(99.4 \%)$ & 1 & 0.56 \\
\hline & & $\mathrm{AA}$ & $1(0.3 \%)$ & $2(0.6 \%)$ & $0.5(0.04-5.54)$ & \\
\hline & \multirow{2}{*}{ Overdominant } & $\mathrm{CC}+\mathrm{AA}$ & $272(91 \%)$ & $265(88.3 \%)$ & 1 & 0.29 \\
\hline & & $\mathrm{CA}$ & $27(9 \%)$ & $35(11.7 \%)$ & $0.75(0.44-1.28)$ & \\
\hline & Log-additive & & & & $0.74(0.45-1.21)$ & 0.22 \\
\hline
\end{tabular}

Table 4. Genotype and allele frequencies of HOTTIP SNPs in cases and controls, and genotype- and allelotype-specific risks. OR, odds ratio; CI, confidence interval; SNPs, single nucleotide polymorphisms.

that the T allele and GT + TT genotype of rs 1899663 in HOTAIR were correlated with the higher GC risk (T vs. $\mathrm{G}, \mathrm{OR}=1.27$; $\mathrm{GT}+\mathrm{TT}$ vs. $\mathrm{GG}, \mathrm{OR}=1.5)$.

The C allele of rs7958904 in HOTAIR was correlated with the increased risk of GC (C vs. G, OR=1.31). Patients carrying the GC or CC genotype of rs7958904 had considerably increased the risk of GC compared to those carrying the GG genotype ( $\mathrm{OR}=1.54$ and $\mathrm{GC}$ vs. $\mathrm{GG}, \mathrm{OR}=1.64$, respectively). In addition, subjects carrying the GC + CC genotype of rs7958904 possessed a meaningful increased risk of GC compared to individuals carrying the GG genotype $(\mathrm{OR}=1.57)$. It has been shown that the HOTAIR rs7958904 CC genotype associates with the higher cervical cancer risk in comparison to the GG/GC genotypes $(\mathrm{OR}=1.57)$. TCGA database revealed that the cervical cancer tissues with the rs7958904 CC genotype had increased the expression of HOTAIR compared to those with GG genotype. Hence, HOTAIR rs7958904 may affect cervical cancer susceptibility by the modulation of CC cell proliferation ${ }^{31}$. It is the possibility of additive roles of genetic and environmental factors with SNPs and understanding gene-gene/gene-environmental interactions are prerequisites for highly effective prevention.

Du et al. demonstrated that the HOTAIR SNP rs4759314 was significantly associated with the increased risk of GC (OR =1.39). The HOXC11 and HOTAIR expression levels in the subjects with AG genotype were much higher than those with AA genotype. In the same vein, the promoter activity of $\mathrm{G}$ allele was more significant than 


\begin{tabular}{|c|c|c|c|c|c|c|c|c|c|c|c|}
\hline & \multicolumn{3}{|l|}{ Gender } & \multicolumn{4}{|c|}{ Tumor origin } & \multicolumn{4}{|l|}{ Pathology } \\
\hline & Male & Female & $p$-value & Cardia & $\begin{array}{l}\text { Cardia/Non- } \\
\text { cardia }\end{array}$ & Non-cardia & $p$-value & $\begin{array}{l}\text { Intestinal-type } \\
\text { GC }\end{array}$ & Diffuse-type GC & Others & $p$-value \\
\hline \multicolumn{3}{|c|}{ rs17720428 } & 0.19 & & & & 0.58 & & & & 0.69 \\
\hline TT & $80(78.4 \%)$ & $22(21.6 \%)$ & & $56(33.3 \%)$ & $3(66.7 \%)$ & $43(42.2 \%)$ & & $48(47.1 \%)$ & $20(19.6 \%)$ & $34(33.3 \%)$ & \\
\hline TG & $109(70.3 \%)$ & $46(29.7 \%)$ & & $78(50.6 \%)$ & $3(1.9 \%)$ & $73(47.4 \%)$ & & $81(52.3 \%)$ & $32(20.6 \%)$ & $42(27.1 \%)$ & \\
\hline GG & $35(81.4 \%)$ & $8(18.6 \%)$ & & $27(62.8 \%)$ & $1(2.3 \%)$ & $15(39.9 \%)$ & & $20(46.5 \%)$ & $7(16.3 \%)$ & $16(37.2 \%)$ & \\
\hline \multicolumn{3}{|c|}{ rs7958904 } & 0.31 & & & & 0.54 & & & & 0.85 \\
\hline GG & $62(77.5 \%)$ & $18(22.5 \%)$ & & $45(56.3 \%)$ & $2(2.5 \%)$ & $33(41.3 \%)$ & & $37(46.2 \%)$ & $16(20 \%)$ & $27(33.8 \%)$ & \\
\hline GC & $110(71 \%)$ & $45(29 \%)$ & & $80(51.9 \%)$ & $2(1.3 \%)$ & $72(46.8 \%)$ & & $80(51.6 \%)$ & $32(20.7 \%)$ & $43(27.7 \%)$ & \\
\hline $\mathrm{CC}$ & $51(79.7 \%)$ & $13(20.3 \%)$ & & $35(54.7 \%)$ & $3(4.7 \%)$ & $26(40.6 \%)$ & & $32(50 \%)$ & $11(17.2 \%)$ & $21(32.8 \%)$ & \\
\hline \multicolumn{3}{|c|}{ rs1899663 } & 0.18 & & & & 0.63 & & & & 0.64 \\
\hline GG & $80(78.4 \%)$ & $22(21.6 \%)$ & & $55(53.9 \%)$ & $3(2.9 \%)$ & $44(43.1 \%)$ & & $48(47.1 \%)$ & $20(19.6 \%)$ & $34(33.3 \%)$ & \\
\hline GT & $108(70.1 \%)$ & 46 (29.9\%) & & $78(51 \%)$ & $3(2 \%)$ & $72(47.1 \%)$ & & $81(52.6 \%)$ & $32(20.8 \%)$ & $42(26.6 \%)$ & \\
\hline TT & $35(81.4 \%)$ & $8(18.6 \%)$ & & $27(62.8 \%)$ & $1(2.3 \%)$ & $15(34.9 \%)$ & & $20(46.5 \%)$ & $7(16.3 \%)$ & $16(37.2 \%)$ & \\
\hline \multicolumn{3}{|c|}{ rs4759314 } & 0.33 & & & & 0.049 & & & & 0.82 \\
\hline $\mathrm{AA}$ & $212(74.1 \%)$ & $74(25.9 \%)$ & & $155(54.4 \%)$ & $5(1.8 \%)$ & $125(43.9 \%)$ & & $141(49.3 \%)$ & 57 (19.9\%) & $88(30.8 \%)$ & \\
\hline AG & $12(85.7 \%)$ & $2(14.3 \%)$ & & $6(42.9 \%)$ & $2(14.3 \%)$ & $6(42.9 \%)$ & & $8(57.1 \%)$ & $2(14.3 \%)$ & $4(28.6 \%)$ & \\
\hline \multicolumn{3}{|c|}{ rs3807598 } & 0.049 & & & & 0.29 & & & & 0.2 \\
\hline CC & $61(67.8 \%)$ & $29(32.2 \%)$ & & $43(48.3 \%)$ & $2(2.3 \%)$ & $44(49.4 \%)$ & & $50(55.6 \%)$ & $19(21.1 \%)$ & $21(23.3 \%)$ & \\
\hline CG & $110(81.5 \%)$ & $25(18.5 \%)$ & & $82(60.7 \%)$ & $3(22.3 \%)$ & $50(37 \%)$ & & $58(43 \%)$ & $27(20 \%)$ & $50(37 \%)$ & \\
\hline GG & $52(70.3 \%)$ & $22(29.7 \%)$ & & $36(48.6 \%)$ & $2(2.7 \%)$ & $36(48.6 \%)$ & & $40(54.1 \%)$ & $13(17.6 \%)$ & $21(28.4 \%)$ & \\
\hline \multicolumn{3}{|c|}{ rs17501292 } & 0.08 & & & & 0.96 & & & & 0.44 \\
\hline TT & $158(76 \%)$ & $50(24 \%)$ & & $110(52.9 \%)$ & $5(2.4 \%)$ & $99(44.7 \%)$ & & $97(46.6 \%)$ & $44(21.2 \%)$ & $67(32.2 \%)$ & \\
\hline TG & $59(77.6 \%)$ & $17(22.4 \%)$ & & $42(56 \%)$ & $2(2.7 \%)$ & $31(41.3 \%)$ & & $43(56.6 \%)$ & $14(18.4 \%)$ & $19(25 \%)$ & \\
\hline GG & $7(50 \%)$ & $7(50 \%)$ & & $7(50 \%)$ & $0(0 \%)$ & $7(50 \%)$ & & $8(57.1 \%)$ & $1(7.1 \%)$ & $5(35.7 \%)$ & \\
\hline \multicolumn{3}{|c|}{ rs1859168 } & 0.28 & & & & 0.74 & & & & 0.63 \\
\hline $\mathrm{CC}$ & $206(76 \%)$ & $65(24 \%)$ & & $145(53.7 \%)$ & $6(2.2 \%)$ & $119(44.1 \%)$ & & $134(49.4 \%)$ & $53(19.6 \%)$ & $84(31 \%)$ & \\
\hline $\mathrm{CA}$ & $17(63 \%)$ & $10(37 \%)$ & & $14(51.9 \%)$ & $1(3.7 \%)$ & $12(44.4 \%)$ & & $14(51.9 \%)$ & $6(22.2 \%)$ & $7(25.9 \%)$ & \\
\hline $\mathrm{AA}$ & $1(100 \%)$ & $0(0 \%)$ & & $1(100 \%)$ & $0(0 \%)$ & $0(0 \%)$ & & $0(0 \%)$ & $0(0 \%)$ & $1(100 \%)$ & \\
\hline
\end{tabular}

Table 5. Subgroup analysis of clinical characteristics for the association of SNPs with GC risk.

that of A allele ${ }^{29}$. Finally, a meta-analysis study by Tao et al. showed that the HOTAIR rs4759314 polymorphism may play a role in GC susceptibility ${ }^{32}$. In this case, all studies were in Chinese populations and therefore could not give an overview of its status in other populations. In contrast, we did not find any significant correlation between the HOTAIR rs4759314 SNPs and GC susceptibility. This may indicate the fact that some HOTAIR risk $\mathrm{SNP}(\mathrm{s})$ may be ancestry-specific; however, this is just a hypothesis and needs to be established, by studying this SNP in other types of cancer in Ardabil and in different (ethnic) population groups suffering from GC.

Only one haplotype in the HOTAIR (GCTA) gene was associated with the risk of GC (OR = 1.31). Studies have shown that different HOTAIR variants (e.g., rs920778, rs7958904, and rs874945) correlate with different cancers, including GC, colorectal cancer, breast cancer, and esophageal cancer ${ }^{33}$. Knockdown of HOTAIR can prevent cell growth of GC, influence cell cycle distribution, and improve P21 and P53 protein levels ${ }^{15}$.

HOTTIP knockdown in GC cells hindered cell proliferation, invasion, and migration. Additionally, HOTTIP down-regulation reduced the expression of homeobox protein Hox-A13 (HOXA13) in cell lines of GC. HOXA13 affected GC cells' HOTTIP-induced malignant phenotypes. Both HOXA13 and HOTTIP were up-regulated in GC tissues than adjacent noncancerous tissues ${ }^{25}$. In the present study, none of the HOTTIP SNPs (i.e., rs3807598, rs17501292, and rs1859168) were associated with the risk of GC. In contrast, Hu et al. showed that HOTTIP rs1859168 A > C notably was associated with a decreased risk of pancreatic cancer (PC) (CC vs. AA: OR=0.71). The $\mathrm{C}$ allele of HOTTIP rs 1859168 could significantly reduce the relative luciferase activity in comparison to the A allele in three PC cell lines. Therefore, the functional rs1859168 A > C polymorphism could reduce the risk of PC by downregulating HOTTIP expression ${ }^{34}$. This discrepancy between the two studies represents the hypothesis that some HOTTIP risk SNPs may be tissue-specific. However, further studies in different cancer cell lines are required to confirm such a hypothesis. In Hepatocellular carcinoma (HCC) patients, HOTTIP rs2071265 was related with an earlier recurrence. The HOTTIP suppression in cancer cell lines of liver decreased the rates of cell invasion and increased chemosensitivity ${ }^{35}$. The interaction of HOTTIP rs17501292 with MALAT1 rs619586 polymorphisms had a decreased impact on the risk of $\operatorname{HCC}(\mathrm{OR}=0.3)^{33}$.

In the present study, although none of the HOTTIP SNPs increased the risk of GC, the SNP-SNP interactions of HOTAIR with HOTTIP were strongly associated with risk of GC. The SNP-SNP interaction of HOTAIR rs17720428 TG with HOTTIP rs1859168 CC increased the risk of GC (OR=1.76). In addition, the SNP-SNP interaction of HOTAIR rs7958904 with HOTTIP rs1859168 increased the risk of GC (rs7958904 GC-rs1859168 $\mathrm{CC}, \mathrm{OR}=1.85$; rs7958904 CC-rs1859168 CC, OR=1.86). Interestingly, the SNP-SNP interaction of HOTAIR 


\begin{tabular}{|c|c|c|c|}
\hline & \multicolumn{3}{|l|}{ Stage } \\
\hline & I, II & III, IV & $p$-value \\
\hline rs17720428 & & & 0.23 \\
\hline TT & $7(7.6 \%)$ & $85(92.4 \%)$ & \\
\hline TG & $5(3.7 \%)$ & $130(96.3 \%)$ & \\
\hline GG & $4(10.3 \%)$ & $35(89.7 \%)$ & \\
\hline rs7958904 & & & 0.08 \\
\hline GG & $6(8.5 \%)$ & $65(91.5 \%)$ & \\
\hline GC & $4(2.9 \%)$ & $132(97.1 \%)$ & \\
\hline $\mathrm{CC}$ & $6(10.3 \%)$ & $52(89.7 \%)$ & \\
\hline rs1899663 & & & 0.23 \\
\hline GG & $7(7.6 \%)$ & $85(92.4 \%)$ & \\
\hline GT & $5(3.7 \%)$ & $130(96.3 \%)$ & \\
\hline TT & $4(10.3 \%)$ & $35(89.7 \%)$ & \\
\hline rs4759314 & & & 0.85 \\
\hline $\mathrm{AA}$ & $15(6 \%)$ & 237 (94\%) & \\
\hline AG & $1(7.1 \%)$ & $13(92.9 \%)$ & \\
\hline rs3807598 & & & 0.98 \\
\hline $\mathrm{CC}$ & $5(6.2 \%)$ & $75(93.8 \%)$ & \\
\hline CG & $7(5.7 \%)$ & $115(94.3 \%)$ & \\
\hline GG & $4(6.3 \%)$ & $59(93.7 \%)$ & \\
\hline rs17501292 & & & 0.44 \\
\hline TT & $9(4.9 \%)$ & $176(95.1 \%)$ & \\
\hline TG & $6(9.2 \%)$ & $59(90.8 \%)$ & \\
\hline GG & $1(7.1 \%)$ & $13(92.9 \%)$ & \\
\hline rs1859168 & & & 0.41 \\
\hline $\mathrm{CC}$ & $16(6.7 \%)$ & $224(93.3 \%)$ & \\
\hline $\mathrm{CA}$ & $0(0 \%)$ & $24(100 \%)$ & \\
\hline $\mathrm{AA}$ & $0(0 \%)$ & $1(100 \%)$ & \\
\hline
\end{tabular}

Table 6. Relationship of clinical stage with HOTAIR/HOTTIP polymorphisms in GC patients.

\begin{tabular}{|c|c|c|c|c|c|c|c|c|}
\hline HOTAIR & rs17720428 & rs7958904 & rs1899663 & rs4759314 & $\begin{array}{l}\text { Frequency in } \\
\text { control group }\end{array}$ & $\begin{array}{l}\text { Frequency in } \\
\text { patients group }\end{array}$ & OR $(95 \% \mathrm{CI})$ & $p$-value \\
\hline & $\mathrm{T}$ & G & G & A & 0.5948 & 0.5281 & 1 & - \\
\hline & G & $\mathrm{C}$ & $\mathrm{T}$ & $\mathrm{A}$ & 0.3432 & 0.4 & $1.31(1.03-1.67)$ & 0.029 \\
\hline & $\mathrm{T}$ & $\mathrm{C}$ & G & $\mathrm{A}$ & 0.0453 & 0.0469 & $1.16(0.67-2.01)$ & 0.61 \\
\hline & $\mathrm{T}$ & $\mathrm{C}$ & G & G & 0.0149 & 0.0233 & $1.72(0.73-4.08)$ & 0.22 \\
\hline & G & $\mathrm{C}$ & $\mathrm{T}$ & G & 0.0018 & - & - & - \\
\hline & G & $\mathrm{C}$ & G & A & - & 0.0017 & - & - \\
\hline \multirow[t]{7}{*}{ HOTTIP } & rs3807598 & rs17501292 & rs1859168 & - & & & & \\
\hline & G & $\mathrm{T}$ & C & - & 0.4843 & 0.4696 & 1 & - \\
\hline & C & $\mathrm{T}$ & C & - & 0.3181 & 0.3121 & $1.01(0.78-1.31)$ & 0.93 \\
\hline & C & G & C & - & 0.1326 & 0.1676 & $1.28(0.92-1.77)$ & 0.14 \\
\hline & C & $\mathrm{T}$ & $\mathrm{A}$ & - & 0.0571 & 0.0444 & $0.77(0.45-1.32)$ & 0.34 \\
\hline & C & G & $\mathrm{A}$ & - & 0.0079 & 0.004 & $0.90(0.16-4.94)$ & \begin{tabular}{|l|}
0.9 \\
\end{tabular} \\
\hline & G & G & C & - & - & 0.0024 & - & - \\
\hline
\end{tabular}

Table 7. Association of the haplotype of HOTAIR/HOTTIP gene with GC risk were calculated using the SNPStats. https://www.snpstats.net/start.htm based on the expectation maximization algorithm.

rs1899663 with HOTTIP rs1859168 strongly increased the risk of GC (rs1899663 GT-rs1859168 CC, OR=4.3; rs1899663 TT-rs1859168 CC, OR=9.37; rs1899663 TT-rs1859168 CA, OR=6.59). To verify the findings and validate the results, further studies in diverse ethnicities and functional analysis are required.

In our research, the stratified analysis of genetic association of the HOTAIR and HOTTIP tagSNPs with clinicopathologic characteristics (such as tumor origin and intestinal-, diffuse-, or indeterminate-types of GC) revealed no significant association in all subgroups. An important problem in GC is that the most GC patients 


\begin{tabular}{|c|c|c|c|c|c|c|}
\hline \multirow{2}{*}{$\begin{array}{l}\text { SNP-SNP interaction } \\
\text { rs17720428 and rs1859168 }\end{array}$} & \multicolumn{2}{|c|}{ SNP Genotype } & \multirow[t]{2}{*}{ Patients } & \multirow[t]{2}{*}{ Controls } & \multirow[t]{2}{*}{ Odds Ratio } & \multirow[t]{2}{*}{$p$-value } \\
\hline & HOTAIR & HOTTIP & & & & \\
\hline & TT & CC & 91 & 122 & 1 & \\
\hline & TT & CA & 10 & 9 & $1.49(0.58-3.81)$ & 0.4 \\
\hline & TT & AA & 1 & 0 & & \\
\hline & TG & $\mathrm{CC}$ & 143 & 109 & $1.76(1.22-2.54)$ & 0.003 \\
\hline & TG & CA & 12 & 21 & $0.77(0.39-1.64)$ & 0.49 \\
\hline & TG & AA & 0 & 1 & & \\
\hline & GG & $\mathrm{CC}$ & 37 & 32 & $1.55(0.9-2.67)$ & 0.11 \\
\hline & GG & $\mathrm{CA}$ & 5 & 5 & $1.34(0.38-4.77)$ & 0.65 \\
\hline & GG & AA & 0 & 1 & & \\
\hline \multirow[t]{10}{*}{ rs7958904 and rs1859168 } & HOTAIR & HOTTIP & & & & \\
\hline & GG & CC & 70 & 103 & 1 & \\
\hline & GG & CA & 9 & 6 & $2.2(0.75-6.47)$ & 0.41 \\
\hline & GG & AA & 1 & 0 & & \\
\hline & GC & $\mathrm{CC}$ & 143 & 114 & $1.85(1.25-2.73)$ & 0.002 \\
\hline & GC & $\mathrm{CA}$ & 12 & 22 & $0.8(0.37-1.73)$ & 0.57 \\
\hline & GC & AA & 0 & 1 & & \\
\hline & $\mathrm{CC}$ & $\mathrm{CC}$ & 57 & 45 & $1.86(1.14-3.06)$ & 0.01 \\
\hline & CC & CA & 6 & 7 & $1.26(0.4-3.91)$ & 0.69 \\
\hline & CC & AA & 0 & 1 & & \\
\hline \multirow[t]{10}{*}{ rs1899663 and rs1859168 } & HOTAIR & HOTTIP & & & & \\
\hline & GG & CC & 37 & 122 & 1 & \\
\hline & GG & $\mathrm{CA}$ & 5 & 9 & $1.83(0.59-5.8)$ & 0.3 \\
\hline & GG & AA & & & & \\
\hline & GT & $\mathrm{CC}$ & 142 & 109 & $4.3(2.75-6.7)$ & $<0.001$ \\
\hline & GT & $\mathrm{CA}$ & 12 & 21 & $1.88(0.85-4.19)$ & 0.12 \\
\hline & GT & AA & 0 & 1 & & \\
\hline & TT & $\mathrm{CC}$ & 91 & 32 & $9.37(5.43-16.18)$ & $<0.001$ \\
\hline & TT & CA & 10 & 5 & $6.59(2.12-20.51)$ & $<0.001$ \\
\hline & TT & AA & 1 & 1 & $3.3(0.2-54.01)$ & 0.38 \\
\hline
\end{tabular}

Table 8. The two-way interaction of HOTAIR and HOTTIP polymorphism in the risk of GC.

\begin{tabular}{|l|l|l|}
\hline & SNP causes miRNA target gain & SNP causes miRNA target loss \\
\hline rs17720428 & $\begin{array}{l}\text { hsa-miR-6513-5p, hsa-miR-450b-3p, hsa-miR-5089-5p, hsa-miR- } \\
769-3 p,\end{array}$ & hsa-miR-5004-3p, \\
\hline rs7958904 & hsa-miR-6721-5p, hsa-miR-1203 & hsa-miR-4750-3p, hsa-miR-615-3p, hsa-miR-6742-5p \\
\hline rs1899663 & - & - \\
\hline rs4759314 & - & - \\
\hline rs3807598 & - & hsa-miR-3115 \\
\hline rs17501292 & hsa-miR-8080 & hsa-miR-1252-5p, hsa-miR-651-3p, hsa-miR-5681a \\
\hline rs1859168 & $\begin{array}{l}\text { hsa-miR-5699-5p, hsa-miR-874-5p, hsa-miR-506-5p, hsa-miR- } \\
216 a-5 p\end{array}$ & - \\
\hline
\end{tabular}

Table 9. The potential impact of each SNP on the establishment or destruction of the miRNA binding site.

are diagnosed at the advanced stage ${ }^{36}$. In the present study, which was confined to Ardabil (a very high-risk area of GC in Northwestern Iran), the $0.8,5.2,45.5$ and $48.5 \%$ of patients were diagnosed at stages I, II, III, and IV, respectively. The frequency of each HOTAIR or HOTTIP tagSNP did not show a significant difference between patients having stage I-II and stage III-IV disease. It might probably be explained by the fact that almost all the patients (94\%) recruited in the study were at the advanced stage (III-IV), having poor prognosis.

The influence of lncRNAs on microRNA function and vice versa is emerging, affecting the gene expression programs. LncRNA tagSNPs can cause or destroy miRNA binding site(s) on the lncRNA. Some LncRNAs act as molecular decoys or sponges of microRNAs, with sequestrating microRNAs favoring the expression of suppressed target mRNAs. Other lncRNAs compete with miRNAs for interacting with shared target mRNAs, causing the derepression of gene expression. They can also be precursors to the production of miRNAs for silencing target mRNAs. In contrast, little is known about the influence of microRNAs on lncRNA function. They can target 
lncRNAs for degradation ${ }^{37,38}$. Here, using bioinformatic analysis, we showed that the HOTAIR rs17720428/ rs7958904 and HOTTIP rs17501292/rs1859168/rs3807598 tagSNPs could lead to miRNA target gain and/or loss. However, for the HOTAIR rs1899663 and rs4759314 tagSNPs, no miRNA target gain or loss was recognized. Among the miRNAs listed in Table 9, for a small number, the functional role has been recently determined to a somewhat large extent in cancer, although not necessarily in GC, including the miR-615-3p, miR-874-5p, miR506-5p, miR-769-3p, miR-1252-5p, and especially miR-216a-5p. For example, miR-615-3p can promote the epithelial mesenchymal transition (EMT) and metastasis of breast cancer by targeting protein interacting with C kinase 1 (PICK1)/TGFBRI axis ${ }^{39}$. MicroRNA-874-mediated inhibition of the major G1/S phase cyclin, cyclin E1 (CCNE1) does not occur in osteosarcomas. It also inhibits tumor metastasis in hepatocellular carcinoma by targeting the $\delta$ opioid receptor (DOR)/epidermal growth factor receptor (EGFR)/extracellular signal-regulated kinase (ERK) pathway ${ }^{40,41}$. MiR-506 inhibits the proliferation and invasion of i) colorectal cancer by targeting ubiquitin-like with plant homeodomain and RING finger domains 1 (UHRF1) via the KISS1/PI3K/NF-kB signaling axis and ii) nasopharyngeal carcinoma by targeting Forkhead box Q1 (FOXQ1), and is also epigenetically silenced in pancreatic cancer ${ }^{42-44}$. During Reoxygenation microRNA-769-3p down-regulates N-myc downstreamregulated gene 1 (NDRG1) and enhances Apoptosis ${ }^{45}$. By targeting miR-1252-5p, the lncRNA AL161431.1 can facilitate cellular proliferation and migration via MAPK signaling in endometrial carcinoma ${ }^{46}$. The function of miR-216a-5p has also been studied in depth in various cancers, playing a role of tumor suppressor. It inhibits the cell proliferation and metastasis by targeting Janus kinase 2 (JAK2)/signal transducer and activator of transcription 3 (STAT3)-mediated EMT process in GC and by targeting p21-activated protein kinase 2 (PAK2) in breast cancer. It also inhibits the cell proliferation and induces apoptosis by targeting tectonic family member 1 (TCTN1) in esophageal squamous cell carcinoma. Moreover, the low expression of miR-216a results in the upregulation of tetraspanin 1 (TSPAN1) that contributes to pancreatic cancer progression via transcriptional regulation of integrin alpha 2 (ITGA2) $)^{47-50}$.

Except for miR-615-3p and miR-1252-5p, which have lost their potential binding sites to HOTAIR (due to rs7958904 polymorphism) and HOTTIP (due to rs17501292 polymorphism), respectively and are thought to be oncogenic, the other four molecules including miR-874-5p, miR-506-5p, miR-769-3p, and miR-216a-5p play the role of tumor suppressors. MiR-615-3p and miR-1252-5p molecules may retain their oncogenic effect due to the loss of their binding site to HOTAIR and HOTTIP, respectively; however, the possible mechanism(s) is unknown and requires functional studies. MiR-769-3p has a potential binding site to HOTAIR due to the rs17720428 polymorphism associated with GC in the present study. Interestingly, all the three molecules miR-874-5p, miR506-5p, and miR-216a-5p have a possible binding site to HOTTIP due to rs 1859168 polymorphism. In the present study, the SNP-SNP interaction of HOTAIR rs1899663 with HOTTIP rs1859168 was strongly associated with GC, which may be due to the destruction of these molecules that are thought to function as tumor suppressors. However, functional studies need to be done to determine if these bindings actually occur and what the role of binding of these molecules to HOTTIP is in the progression to GC. These studies should be performed in the presence of HOTTIP rs1859168 tagSNPs by controlling the presence of HOTAIR rs1899663 polymorphism.

Taking altogether, we showed that HOTAIR rs17720428, rs7958904, and rs1899663 tagSNPs and their interactions with the HOTTIP rs 1859168 polymorphism were significantly associated with GC risk. Specifically, novel SNP-SNP interactions between HOTAIR and HOTTIP tagSNPs have a larger impact than individual SNP effects on GC risk, thereby providing us with valuable information to reveal potential biological mechanisms for developing GC.

\section{Materials and methods}

Study subjects. A hospital-based case-control study, from October 2017 to February 2019, was conducted. A total of 300 cases were selected from patients undergoing endoscopic examination in the Imam Khomeini Hospital in the Ardabil. One control was sought for each case, frequency matched to the case group by 5 -year age groups and gender. The controls were randomly selected from subjects who received routine physical examinations in the same hospital and had no self-reported history of cancer at any site. According to histopathologic and endoscopic results, gastroduodenal disease was diagnosed. GC diagnoses were categorized by anatomic subsites based on the International Classification of Diseases, $10^{\text {th }}$ Revision (ICD-10) as cardia (ICD-10 code C16.0) and non-cardia (ICD-10 codes C16.1-C16.9, involving unspecified and overlapping subsites) ${ }^{51}$. According to the classification of Lauren, histologic subtypes were assessed as diffuse-type, intestinal-type, and other/unspecified histologies ${ }^{52}$. Finally, the AJCC 8th TNM staging system for GC was considered, showing an improved efficiency in GC prognosis ${ }^{53}$. The study was conducted on the basis of ethical principles of human research expressed in the 1975 Declaration of Helsinki. All participants signed an informed consent form. This study was approved by the Ethics Committee of the National Institute for Medical Research Development (NIMAD)/ IR.NIMAD. REC.1396.097.

SNP selection and genotyping. The data of genetic polymorphism from the entire sequence of lncRNAs was achieved from the dbSNP database (https://www.ncbi.nlm.nih.gov/projects/SNP/). The lncRNA HOTTIP gene sequences were downloaded by the 1000 Genomes Browser (https://www.ncbi.nlm.nih.gov/variation/tools /1000genomes/) after enlarging $2 \mathrm{~kb}$ of upstream and downstream flanking sequences of the gene. The selection criteria were: (i) linkage disequilibrium (LD) $r 2$ lower than 0.8 , (ii) minor allele frequency (MAF) higher than 0.05 , and (iii) the $p$-value of Hardy-Weinberg equilibrium (HWE) higher than 0.05 . Seven eligible tagSNPs were chosen involving four SNPs for HOTAIR (i.e., rs17720428, rs7958904, rs1899663, and rs4759314) and three SNPs for HOTTIP (i.e., rs3807598, rs17501292, and rs17501292) eventually included in the final analysis. From each participant, venous blood samples were taken into an ethylenediaminetetraacetic acid (EDTA)-containing tube and were stored at $-80^{\circ} \mathrm{C}$. Using QIAamp DNA blood mini kit (QIAGEN, Germany), genomic DNA was 
extracted from $200 \mu \mathrm{L}$ peripheral blood samples as previously described ${ }^{54}$. All samples were genotyped by the Infinium HTS platform according to the standard protocol (https:/www.illumina.com/Documents/products/ workflows/workflow_infinium_ii.pdf) with a customized Illumina Infinium GSA BeadChip_a robust, highquality assay. This SNP microarray uses known nucleotide sequences as probes to hybridize with the tested DNA sequences, allowing a qualitative and quantitative SNP analysis. Data quality control was performed using Genome Studio. The call rate cut-off was $98 \%$ as it an off-the-shelf array.

Statistical and bioinformatic analysis. Genotyping results of SNPs were evaluated for significant departure from Hardy-Weinberg equilibrium. Using Pearson chi-square test or Fisher's exact probability (for categorical variables), the variations in frequency distribution of genotypes and demographic characteristics were assessed. The association strength was calculated applying odds ratios (ORs) and $95 \%$ confidence intervals (CIs). All genetic models were evaluated, including dominant, recessive, co-dominant, over dominant, and log additive models of inheritance for seven SNPs. Each model provides different assumptions regarding the genetic effect. Using the SNPStats (https:/www.snpstats.net/start.htm), haplotype frequencies were obtained for HOTAIR and HOTTIP according to the expectation maximization algorithm. The pairwise interactions of lncRNA SNP-SNP were calculated. Statistical analyses were done by SPSS version 19.0 (IBM, Chicago, USA). The correlations between every genetic variant and clinical features of GC were investigated. The statistical tests were two-sided; $p<0.05$ was assumed statistically significant. The potential impact of each SNP on the establishment or destruction of the miRNA binding site was analyzed using the lncRNASNP2 database ${ }^{55}$.

\section{Data availability}

The data that support the findings of this study are available from the corresponding author upon reasonable request.

Received: 17 June 2020; Accepted: 22 September 2020

Published online: 07 October 2020

\section{References}

1. Abdi, E., Latifi-Navid, S., Zahri, S., Yazdanbod, A. \& Pourfarzi, F. Risk factors predisposing to cardia gastric adenocarcinoma: Insights and new perspectives. Cancer Med. https://doi.org/10.1002/cam4.2497 (2019).

2. Bakhti, S. Z., Latifi-Navid, S. \& Safaralizadeh, R. Helicobacter pylori-related risk predictors of gastric cancer: The latest models, challenges, and future prospects. Cancer Med. https://doi.org/10.1002/cam4.3068 (2020).

3. Abdi, E., Latifi-Navid, S., Latifi-Navid, H. \& Safarnejad, B. Helicobacter pylori vacuolating cytotoxin genotypes and preneoplastic lesions or gastric cancer risk: a meta-analysis. J. Gastroenterol. Hepatol. 31, 734-744. https://doi.org/10.1111/jgh.13256 (2016).

4. Bray, F. et al. Global cancer statistics 2018: GLOBOCAN estimates of incidence and mortality worldwide for 36 cancers in 185 countries. CA Cancer J. Clin. 68, 394-424. https://doi.org/10.3322/caac.21492 (2018).

5. Correa, P. Human gastric carcinogenesis: a multistep and multifactorial process-first American Cancer Society award lecture on cancer epidemiology and prevention. Can. Res. 52, 6735-6740 (1992).

6. Wu, M.-S., Chen, C.-J. \& Lin, J.-T. Host-environment interactions: their impact on progression from gastric inflammation to carcinogenesis and on development of new approaches to prevent and treat gastric cancer. Cancer Epidemiol. Prevent. Biomark. 14, 1878-1882 (2005).

7. Sud, A., Kinnersley, B. \& Houlston, R. S. Genome-wide association studies of cancer: current insights and future perspectives. Nat. Rev. Cancer 17, 692-704 (2017).

8. Pennisi, E. (American Association for the Advancement of Science, 2011).

9. Kumar, V., Wijmenga, C. \& Withoff, S. in Seminars in Immunopathology. 567-580 (Springer).

10. Ma, L., Bajic, V. B. \& Zhang, Z. On the classification of long non-coding RNAs. RNA Biol. 10, 924-933 (2013).

11. Fatica, A. \& Bozzoni, I. Long non-coding RNAs: new players in cell differentiation and development. Nat. Rev. Genet. 15, 7-21 (2014).

12. Lin, R., Maeda, S., Ca, L., Karin, M. \& Edgington, T. A large noncoding RNA is a marker for murine hepatocellular carcinomas and a spectrum of human carcinomas. Oncogene 26, 851-858 (2007).

13. Li, T. et al. Expression and clinicopathological significance of the lncRNA HOXA11-AS in colorectal cancer. Oncol. Lett. 12, 4155-4160 (2016).

14. Pibouin, L. et al. Cloning of the mRNA of overexpression in colon carcinoma-1: a sequence overexpressed in a subset of colon carcinomas. Cancer Genet. Cytogenet. 133, 55-60 (2002).

15. Xu, Z. et al. The association of HOTAIR with the diagnosis and prognosis of gastric cancer and its effect on the proliferation of gastric cancer cells. Can. J. Gastroenterol. Hepatol.2019 (2019).

16. Elsayed, E. T., Salem, P. E., Darwish, A. M. \& Fayed, H. M. Plasma long non-coding RNA HOTAIR as a potential biomarker for gastric cancer. Int. J. Biol. Mark. 33, 528-533 (2018).

17. Kazemzadeh, M. et al. LOC100287225, novel long intergenic non-coding RNA, misregulates in colorectal cancer. Cancer Biomark. 16, 499-505 (2016).

18. Zou, Z. et al. Long intergenic non-coding RNA 00324 promotes gastric cancer cell proliferation via binding with HuR and stabilizing FAM83B expression. Cell Death Dis. 9, 1-14 (2018).

19. Lin, M.-T., Song, H.-J. \& Ding, X.-Y. Long non-coding RNAs involved in metastasis of gastric cancer. World J. Gastroenterol. 24, 3724 (2018).

20. Abdeahad, H. et al. The prognostic potential of long noncoding RNA HOTAIR expression in human digestive system carcinomas: A meta-analysis. J. Cell Physiol. 234, 10926-10933. https://doi.org/10.1002/jcp.27918 (2019).

21. Liu, H. et al. Long noncoding RNA LINC00941 as a potential biomarker promotes the proliferation and metastasis of gastric cancer. Front. Genet. 10, 5 (2019).

22. Tang, Q. \& Hann, S. S. HOTAIR: an oncogenic long non-coding RNA in human cancer. Cell Physiol. Biochem. 47, 893-913. https ://doi.org/10.1159/000490131 (2018).

23. Lian, Y. et al. HOTTIP: a critical oncogenic long non-coding RNA in human cancers. Mol. BioSyst. 12, 3247-3253 (2016).

24. Wu, D. C. et al. Reprogramming antagonizes the oncogenicity of HOXA13-long noncoding RNA HOTTIP axis in gastric cancer cells. Stem Cells 35, 2115-2128. https://doi.org/10.1002/stem.2674 (2017).

25. Chang, S. et al. HOTTIP and HOXA13 are oncogenes associated with gastric cancer progression. Oncol. Rep. 35, 3577-3585 (2016). 
26. Li, D. et al. Long noncoding RNA HULC modulates the phosphorylation of YB-1 through serving as a scaffold of extracellular signal-regulated kinase and YB-1 to enhance hepatocarcinogenesis. Hepatology 65, 1612-1627 (2017).

27. Su, J. et al. Long noncoding RNA BLACAT1 indicates a poor prognosis of colorectal cancer and affects cell proliferation by epigenetically silencing of p15. Cell Death Dis. 8, e2665-e2665 (2017).

28. Yang, C. et al. (2015).

29. Du, M. et al. The association analysis of IncRNA HOTAIR genetic variants and gastric cancer risk in a Chinese population. Oncotarget 6, 31255 (2015).

30. Prensner, J. R. \& Chinnaiyan, A. M. The emergence of lncRNAs in cancer biology. Cancer Discov. 1, 391-407 (2011).

31. Jin, H. et al. HOTAIR rs7958904 polymorphism is associated with increased cervical cancer risk in a Chinese population. Sci. Rep. 7, 1-6 (2017).

32. Tao, T. et al. Association between LncRNA HOTAIR rs4759314 A> G polymorphism and cancer risk: a meta-analysis based on 5525 cases and 6657 controls in Chinese populations. Int. J. Clin. Exp. Med. 9, 12780-12787 (2016).

33. Wang, B.-G. et al. Association of twelve polymorphisms in three onco-lncRNA genes with hepatocellular cancer risk and prognosis: a case-control study. World J. Gastroenterol. 24, 2482 (2018).

34. Hu, P. et al. rs1859168 A $>$ C polymorphism regulates HOTTIP expression and reduces risk of pancreatic cancer in a Chinese population. World J. Surg. Oncol. 15, 155 (2017).

35. Wu, L. et al. Long noncoding RNA HOTTIP expression predicts tumor recurrence in hepatocellular carcinoma patients following liver transplantation. Hepatobiliary Surg. Nutr. 7, 429 (2018).

36. Takahashi, T., Saikawa, Y. \& Kitagawa, Y. Gastric cancer: current status of diagnosis and treatment. Cancers 5, 48-63. https://doi. org/10.3390/cancers5010048 (2013).

37. Yoon, J. H., Abdelmohsen, K. \& Gorospe, M. Functional interactions among microRNAs and long noncoding RNAs. Semin. Cell Dev. Biol. 34, 9-14. https://doi.org/10.1016/j.semcdb.2014.05.015 (2014).

38. Fernandes, J. C. R., Acuna, S. M., Aoki, J. I., Floeter-Winter, L. M. \& Muxel, S. M. Long non-coding RNAs in the regulation of gene expression: physiology and disease. Non-coding RNA https://doi.org/10.3390/ncrna5010017 (2019).

39. Lei, B. et al. miR-615-3p promotes the epithelial-mesenchymal transition and metastasis of breast cancer by targeting PICK1/ TGFBRI axis. J. Exp. Clin. Cancer Res. CR 39, 71. https://doi.org/10.1186/s13046-020-01571-5 (2020).

40. Ghosh, T. et al. MicroRNA-874-mediated inhibition of the major G1/S phase cyclin, CCNE1, is lost in osteosarcomas. J. Biol. Chem. 292, 21264-21281. https://doi.org/10.1074/jbc.M117.808287 (2017).

41. Zhang, Y. et al. microRNA-874 suppresses tumor proliferation and metastasis in hepatocellular carcinoma by targeting the DOR/ EGFR/ERK pathway. Cell Death Dis. 9, 130. https://doi.org/10.1038/s41419-017-0131-3 (2018).

42. Lin, Y. et al. MiR-506 targets UHRF1 to inhibit colorectal cancer proliferation and invasion via the KISS1/PI3K/NF-kappaB signaling axis. Front. Cell Dev. Biol. 7, 266. https://doi.org/10.3389/fcell.2019.00266 (2019).

43. Zhang, Z. et al. MiR-506 suppresses tumor proliferation and invasion by targeting FOXQ1 in nasopharyngeal carcinoma. PLoS ONE 10, e0122851. https://doi.org/10.1371/journal.pone.0122851 (2015).

44. Li, J. et al. Downregulated miR-506 expression facilitates pancreatic cancer progression and chemoresistance via SPHK1/Akt/ NF-kappaB signaling. Oncogene 35, 5501-5514. https://doi.org/10.1038/onc.2016.90 (2016).

45. Luo, E. C. et al. MicroRNA-769-3p down-regulates NDRG1 and enhances apoptosis in MCF-7 cells during reoxygenation. Sci. Rep. 4, 5908. https://doi.org/10.1038/srep05908 (2014).

46. Gu, Z. R. \& Liu, W. The LncRNA AL1614311 targets miR-1252-5p and facilitates cellular proliferation and migration via MAPK signaling in endometrial carcinoma. Eur. Rev. Med. Pharmacol. Sci. 24, 2294-2302. https://doi.org/10.26355/eurrev_202003_20495 (2020).

47. Tao, Y. et al. MicroRNA-216a inhibits the metastasis of gastric cancer cells by targeting JAK2/STAT3-mediated EMT process. Oncotarget 8, 88870-88881. https://doi.org/10.18632/oncotarget.21488 (2017).

48. Wang, S. et al. miR-216a-mediated upregulation of TSPAN1 contributes to pancreatic cancer progression via transcriptional regulation of ITGA2. Am. J. Cancer Res. 10, 1115-1129 (2020).

49. Chai, L. \& Yang, G. MiR-216a-5p targets TCTN1 to inhibit cell proliferation and induce apoptosis in esophageal squamous cell carcinoma. Cell. Mol. Biol. Lett. 24, 46. https://doi.org/10.1186/s11658-019-0166-9 (2019).

50. Zhang, Y. et al. MiR-216a-5p act as a tumor suppressor, regulating the cell proliferation and metastasis by targeting PAK2 in breast cancer. Eur. Rev. Med. Pharmacol. Sci. 23, 2469-2475. https://doi.org/10.26355/eurrev_201903_17394 (2019).

51. in International statistical classification of diseases and related health problems. (ed WHO Library Cataloguing-in-Publication Data.) (2016).

52. Lauren, P. The two histological main types of gastric carcinoma: diffuse and so-called intestinal-type carcinoma: an attempt at a histo-clinical classification. Acta Pathol. Microbiol. Scand. 64, 31-49 (1965).

53. Amin, M. B. et al. The Eighth Edition AJCC Cancer Staging Manual: Continuing to build a bridge from a population-based to a more "personalized" approach to cancer staging. CA Cancer J. Clin. 67, 93-99. https://doi.org/10.3322/caac.21388 (2017).

54. Warton, K., Graham, L. J., Yuwono, N. \& Samimi, G. Comparison of 4 commercial kits for the extraction of circulating DNA from plasma. Cancer Genetics 228-229, 143-150. https://doi.org/10.1016/j.cancergen.2018.02.004 (2018).

55. Miao, Y. R., Liu, W., Zhang, Q. \& Guo, A. Y. IncRNASNP2: an updated database of functional SNPs and mutations in human and mouse lncRNAs. Nucleic Acids Res. 46, D276-D280. https://doi.org/10.1093/nar/gkx1004 (2018).

\section{Acknowledgements}

We would like to thank all patients who participated in this study and the clinical staff and research nurses of Aras Clinic at Imam Khomeini Hospital, particularly Farideh Feizi and Nasrin Fouladi. This study was supported by the National Institute for Medical Research Development (NIMAD) Grant No. 958117, Tehran, Iran. The supporter had no role in study design, data collection and analysis, decision to publish, or preparation of the manuscript.

\section{Author contributions}

S.L-N. provided direction in the preparation of the manuscript; E.A. wrote the first draft of manuscript; V.K-O. and B.M. analyzed data; S.L-N., F.P., and A.Y. extracted data; S.L-N., S.Z., A.Y., V.K-O., and F.P. discussed and revised the manuscript; E.A., A.Y., F.P., and S.Z. managed the references; S.L-N. approved the version to be published.

\section{Competing interests}

The authors declare no competing interests. 


\section{Additional information}

Correspondence and requests for materials should be addressed to S.L.-N.

Reprints and permissions information is available at www.nature.com/reprints.

Publisher's note Springer Nature remains neutral with regard to jurisdictional claims in published maps and institutional affiliations.

(c) (1) Open Access This article is licensed under a Creative Commons Attribution 4.0 International License, which permits use, sharing, adaptation, distribution and reproduction in any medium or format, as long as you give appropriate credit to the original author(s) and the source, provide a link to the Creative Commons licence, and indicate if changes were made. The images or other third party material in this article are included in the article's Creative Commons licence, unless indicated otherwise in a credit line to the material. If material is not included in the article's Creative Commons licence and your intended use is not permitted by statutory regulation or exceeds the permitted use, you will need to obtain permission directly from the copyright holder. To view a copy of this licence, visit http://creativecommons.org/licenses/by/4.0/.

(c) The Author(s) 2020 